platform. CanPath holds data and biosamples on more than 330,000 participants from five regional cohorts representing British Columbia, Alberta, Ontario, Quebec, Nova Scotia, New Brunswick, Prince Edward Island, and Newfoundland and Labrador. A sixth cohort representing Manitoba has begun recruitment and Saskatchewan is in the planning stages.

Objectives To examine the genetic, environmental and lifestyle factors that may influence the development of cancer and chronic disease.

Methods A standardized baseline questionnaire was implemented across CanPath between 2009-2015. Participants also provided biosamples including blood, saliva, urine, and toenails, and non-invasive physical measures (height, weight, hip and waist circumference, body composition, and blood pressure). Subsequently, the first follow-up questionnaire was implemented between 2016-2018. Data from supplementary questionnaires are also available from regional cohorts.

Results CanPath holds a harmonized dataset with 1,477 variables including demographics, history of cancer and other chronic disease, lifestyle and health behaviours, and physical measures. Variables of particular relevance to occupational health research include geographic location, sleep, job title, occupational history, work status, and work schedule. In addition, $>150,000$ participants provided blood and/or other biosamples.

Conclusions CanPath represents a powerful tool for population health research. The survey data and biosamples are available to researchers for future use to gain a more in-depth understanding of the causes and consequences related to occupational health among Canadian residents.

\section{P-16 EFFECTIVENESS OF THE BRAZILIAN VERSION OF THE DANGEROUS DECIBELS PROGRAM FOR WORKERS}

${ }^{1}$ Adriana Lacerda, Luciana Bramatti, Jair Mendes Marques, David Welch, Reddy Reddy, Claudia Giglio de Oliveira Gonçalves. 'Universidade Tuiuti do Parana, Brazil

\subsection{6/OEM-2021-EPI.165}

Introduction Noise-induced hearing loss can be avoided by taking preventive measures.

Objective To assess the effectiveness of the Brazilian version of the Dangerous Decibels ${ }^{\circledR}$ program for noise-exposed workers, using the ecological model as an educational intervention plan.

Method Randomized interventional study with a quantitative, experimental trial design, conducted at a meatpacking company. The participants were divided into two groups - the first one $(n=132$, divided into 6 subgroups) received the Dangerous Decibels ${ }^{\circledR}$ educational intervention (DDEI) adapted to workers (REDDY et al., 2017), while the second group ( $n=138$, divided into 5 subgroups) received a conventional educational intervention (CEI). The interventions lasted 50 minutes. The Hearing Protection Assessment Questionnaire (HPA-5) was administered before and after the interventions. The five dimensions (attitude, behavior, knowledge, supports, and barriers) were compared using the Student's t-test for paired data $(<0.05)$.

Results After the DDEI training, workers improved significantly in barriers, supports, knowledge, attitudes, and behavior around noise. For knowledge, attitudes and behavior, the improvement was greater for those trained with the DDEI than the CEI.
Conclusions The Brazilian version of the Dangerous Decibels ${ }^{\circledR}$ program for noise-exposed workers was effective, influencing positively the factors at different levels of the ecological model.

\section{P-18 SEX AND GENDER DIFFERENCES IN OCCUPATIONAL HAZARD EXPOSURES: A SCOPING REVIEW OF LITERATURE FROM THE LAST 10 YEARS}

${ }^{1}$ Aviroop Biswas, Shireen Harbin, Emma Irvin, Heather Johnston, Momtaz Begum, Maggie Tiong, Dorothy Apedaile, Mieke Koehoorn, Peter Smith. 'Institute for Work \& Health, Canada

\subsection{6/OEM-2021-EPI.166}

Introduction Comparative research on sex and/or gender differences in occupational hazard exposures is necessary for effective work injury and illness prevention strategies that integrate individual and social context in their design, especially as women make up half of the labour force in high-income countries.

Objective To summarize the peer-reviewed literature on exposure differences to occupational hazards between men and women, across occupations and within the same occupation.

Methods A scoping review was conducted on studies from 2009 to 2019, from 8 databases. Studies were required to quantify the exposure of men and women to an occupational hazard. The analysis of hazard exposure differences within the same occupations was based on whether studies stratified or matched their results by occupation for men and women, or mentioned in the article. Studies were not limited by language or study design.

Results Fifty-eight studies met our inclusion criteria. Of these, 30 studies were on physical hazards, 38 studies on psychosocial hazards, 5 studies on biological hazards, and 17 studies on chemical hazards. The majority of studies reported that men were exposed to noise, vibration, radiation, physical work, biomechanical and chemical hazards; while women were exposed to wet work, bullying and discrimination, work stress, and biological agents. Within the same occupations, men were more likely to be exposed to physical hazards, with the exception of women in healthcare occupations and prolonged standing exposure. Women compared to men in the same occupations were more likely to experience harassment, while men compared to women in the same occupations reported higher stress. Men reported more exposure to hazardous chemicals in the same occupations as women.

Conclusions Men and women have different exposures to occupational hazards, and these differences are not solely due to the gendered distribution of the labour foce by occupation. Future research is needed to explain the reasons for sex/gender inequalities and differences in exposures within the same occupations.

\section{P-25 ASSESSMENT OF LIGHTING INTENSITY AT WORKSTATIONS AND INCIDENCE OF SHOULDER PAIN AMONG ELECTRONIC MANUFACTURING WORKERS}

${ }^{1}$ Sunisa Chaiklieng. 'Faculty of Public Health Khon Kaen University, Thailand

10.1136/OEM-2021-EPI.167 
Introduction Electronic assembly manufacturing has a working process which includes machine operation, assembly and fine work inspection of products with a microscope.

Objectives This study aimed to assess lighting intensity at workstations compared to the standards and the incidence of shoulder pain among electronic assembly workers.

Methods There were 167 electronic workers in one site of electronic manufacturing. The measurement of lighting intensity was performed with a lux meter at four types of job function, i.e. machine operation, assembly of parts, and two inspection types of moderate fine work and very fine products. The quarterly incidence of shoulder pain was calculated from monthly follow-up.

Results Insufficient lighting intensity was found in the highest proportion at the microscope station of lighting intensity zone 1 (eye-focusing zone) at $>2,000-5,000$

lux $(81.12 \%)$, followed by the inspection with a monitor/ profile projector $(64.29 \%)$, and lighting intensity zone 1 at $<1,000$ lux (57.89\%). The insufficient light was mostly identified within an arm's length of the worker (zone 2) and outside an arm's length (zone 3). The three-month incidence of shoulder pain was $85.3 \%$ and it was found that all workers had experienced shoulder pain at the fourmonth follow-up.

Conclusion This lighting intensity measurement method is suitable for identifying problems of the working environment of inspection stations, which is useful for further implementation. The high incidence of shoulder pain suggests that electronic assembly workers should be aware of the need to take frequently short breaks from eye-focusing work and stretch muscles for prevention of eye fatigue and shoulder pain.

\section{P-28 THE DIAGNOSTIC AND HEALTH SERVICE PROBLEMS OF DISTINGUISHING TB AND SILICOSIS CLINICALLY AND RADIOLOGICALLY AMONG EX-GOLD MINERS IN LESOTHO}

'Botembetume Maboso, Rodney Ehrlich. 'University of Cape Town, South Africa

\subsection{6/OEM-2021-EPI.168}

Background Between January 2017 and November 2018, 2678 former gold miners from South African mines were examined and sputum screened with GeneExpert at Mafeteng Occupational Health Service Centre in Lesotho. Mean duration since last exposure was 12 years. A high prevalence of silicosis (42.5\%), HIV (30.7\%) and tuberculosis (active TB $6.8 \%$, history of past TB treatment $53.4 \%$ ) was found. Of the TB cases, 54,7\% were symptom screen negative (had no TB symptoms in WHO Guidelines), and 9,3\% had normal chest $\mathrm{x}$-rays. In total, $60 \%$ of the sample had lung features on $\mathrm{x}$ ray read as either silicosis, silicotuberculosis, or tuberculosis. It was observed that many silicosis cases had been repeatedly treated for $\mathrm{TB}$, and some even documented as MDR-TB, on the basis of $\mathrm{x}$-ray alone, even when bacteriologic tests gave negative results.

Objectives To illustrate the challenges in diagnosing active TB in this setting, including distinguishing pulmonary TB (past and current) from pneumoconiosis (silicosis, CWP, asbestosis).

Methods Several cases will be discussed. For each case, a complete medical scenario is described, including occupational history, past and current medical history, physical examination, radiological and laboratory findings.
Results

The following were noted (1) Poor agreement between radiological findings and clinical presentation; (2) Poor agreement between radiographs suggestive of TB and positive GeneExpert sputum results; and (3) Similarity of clinical and radiological presentation of silicosis and tuberculosis.

Conclusion TB screening of former gold and other miners needs to take these diagnostic challenges into account. There should be systematic digital record keeping of chest $\mathrm{x}$-rays. Records, including those from the mines, should be accessible to all healthcare providers who attend to former miners in the community. Training on occupational lung disease with emphasis on pneumoconiosis and $\mathrm{TB}$ is needed for all community healthcare providers.

\section{P-29 REGISTERED HEALTH PROBLEMS AND DEMOGRAPHIC PROFILE OF INTEGRATED TEXTILE FACTORY WORKERS IN ETHIOPIA: A CROSS-SECTIONAL STUDY}

${ }^{1}$ Yifokire Zele, Bente E Moen, Magne Bråtveit, Wakgari Deressa, Abera Kumie. 'University, Ethiopia

\subsection{6/OEM-2021-EPI.169}

Background Integrated textile and garment factories are growing in low and middle-income countries, where workers expose to workplace hazards. However, workers' health condition in the sector is inadequately investigated.

Objective This study describes the magnitude of registered health problems and associated work-related and personal factors among workers in integrated textile factories in Ethiopia.

Methods Institution-based cross-sectional study design was employed. A one-year recording of worker's clinical diagnoses (between March 2016 and February 2017) were gathered from the respective factory clinics of three integrated textile factories. Clinical diagnosis data on various diseases were obtained when factory workers visit the clinic for feeling unwell or ill. Sociodemographic characteristics and workrelated information were obtained from the factory's human resource departments. Sociodemographics and clinical diagnosis statuses of 7992 workers were analyzed. The association between diagnoses and workplace factors (textile production, garment production and support process) and personal factors (age, gender and educational status) were studied using logistic regression analyses.

Results The average workers' age and years of service were 40 years and 11 years respectively. 4778 (60\%) of workers, were female. $66 \%$ of the workers $(n=5276)$ were diagnosed with one or more types of diseases among the 27,320 clinical diagnoses in total. This caused 16,993 working days absence due to sick leave. Respiratory diseases (34\%) and musculoskeletal disorders (29\%) were the most prevalent diagnoses; while injuries caused the highest number of days of work absence. Work department, gender and educational status were the variables that were statistically significantly associated with higher prevalence of disease groups.

Conclusions About two-thirds of the integrated textile factory workers were diagnosed with different types of diseases. The textile and garment production department workers were more affected than the support process workers, indicating that some diseases may be related to workplace exposure. Further study should investigate on rare chronic diseases such as 